\title{
Confocal Laser Endomicroscopy in the Management of IBD: Clearly Superior?
}

\author{
Alastair J. M. Watson ${ }^{1}$ \\ Published online: 10 May 2016 \\ (c) Springer Science+Business Media New York 2016
}

The intestinal epithelium forms a barrier against the entry of toxins, antigens and microbes from the environment. The epithelial cell itself, the tight junctions between the epithelial cells, and the mucus covering the epithelium together with antimicrobial peptides in the mucus all contribute to the barrier function of the epithelium. Abnormalities of these three elements are now well documented to substantially contribute to the pathogenesis of inflammatory bowel disease (IBD). Recently, a fourth component to this barrier has come to light; understanding this component requires review of the cell dynamics of the intestinal epithelium. The intestinal epithelium arises from stem cells located at the base of crypts, which then migrate up the crypt-villus axis in the case of the small intestine or to the surface of the colon from where they are shed. This shedding process could potentially create a gap in the epithelial monolayer thereby compromising the integrity of the epithelial barrier. In health, the integrity of the barrier is maintained during shedding by an overall redistribution of tight junctional proteins around the epithelial cell plugging the gap created by the shedding cell. When the intestine is inflamed, cytokines such as tumor necrosis factor (TNF)- $\alpha$ induced apoptosis in the epithelium, greatly increasing the rate of epithelial shedding. In these circumstances adjacent epithelial cells are shed, creating gaps that are too large to be sealed by a redistribution of tight junction-associated proteins [1].

Alastair J. M. Watson

alastair.watson@uea.ac.uk

1 Norwich Medical School, University of East Anglia, Rm 2.14 Elizabeth Fry Building, Norwich Research Park, Norwich NR4 7TJ, England, UK
Confocal microscopy in animal models and confocal endomicroscopy in humans have been essential tools for the study of epithelial cell shedding. Confocal microscopy differs from conventional microscopy in that its optics are arranged so that only elements in the optical plane of focus contribute to the image. Since light from above and below the focal plane is excluded, a sharply focused image is generated. This is a huge advantage, as physical sectioning of the tissue is no longer required to form a focused image. As a consequence, highly focused images can be formed within translucent tissue. When incorporated into an endoscope, histological images below the surface of the epithelium can be formed without the need for conventional pinch biopsies or subsequent sectioning, enabling histological examination of the intestine in real time during the endoscopic procedure. Since fluorescence is required to form a confocal image, a fluorescent contrast agent must be injected or applied to the surface of the intestine immediately before gathering the confocal image.

When confocal laser endomicroscopy (CLE) was introduced in the early 2000s, it was first used for the examination of dysplastic and neoplastic tissue in IBD and Barrett's esophagus. It was then discovered that intravenous fluorescein could enable the detection of shedding epithelial cells and gaps in the epithelial monolayer. Furthermore, areas of loss of barrier function could be detected, which appeared as intravenous fluorescein escaping into the intestinal lumen. Using this type of endoscopic imaging, a number of studies have reported that excessive epithelial cell shedding and barrier loss as defined by escape of fluorescein into the lumen predicts future relapse in IBD patients who are in remission at the time of CLE [2, 3]. Of interest, these results mirror earlier techniques using sugar absorption studies to measure the intestinal barrier which also predict relapse of IBD [4]. 
Now that the principle has been established that fluorescein-based CLE can predict relapse of IBD, further studies need to undertaken to validate how to quantify and interpret CLE images. Previous studies have employed a number of approaches to quantifying abnormalities in CLE images [3]. In this issue of Digestive Diseases and Sciences, Liu et al. [5] address the issue of whether the density of gaps detected with CLE correlates with quantitative analysis of activated caspase in conventional mucosal biopsies from patients. The basis of this study is that epithelial cells undergoing apoptotic cell death during the shedding process activate an enzyme termed caspase 3 while cells undergoing necrosis or pyroptosis during shedding activate caspase 1 [6]. The investigators recruited 13 patients with either Crohn's disease, ulcerative colitis or diarrhea-predominant irritable bowel disease (IBS) and 12 control patients who were undergoing conventional colonoscopy either for evaluation of symptoms or colorectal cancer screening. During colonoscopy, the subjects also underwent probe-based CLE in which a confocal instrument was passed through the biopsy channel of the colonoscope. Each subject received an intravenous injection of $5 \mathrm{ml}$ of $10 \%$ fluorescein prior to image collection. Images were collected from five sites within $10 \mathrm{~cm}$ of the terminal ileum. Two reviewers blinded to the status of the subjects undertook review of the images after completion of the colonoscopy. The number of epithelial cells and gaps located on the villi were counted manually. Gap density was then calculated as the number of epithelial gaps per 1000 epithelial cells. After imaging was complete, pinch biopsies were collected as close to the imaging site as possible. Biopsy of visible lesions was avoided. Immunohistochemistry for activated caspase 1 and 3 was undertaken. Once more 2 reviewers blinded to the subject status counted the density of cells with activated caspase 1 or 3 .

The gap density from CLE images was 10/1000 cells in control patients compared to $32 / 1000$ cells in IBS patients and 90/1000 in IBD patients with a density in IBD and IBS patients combined of 33/1000. The caspase-positive cell density (caspase 1 and 3 combined) was 26/1000 cells against 13/1000 in control subjects. There were more cells that were positive for caspase 1 than caspase 3 . Indeed, the number of caspase 3 positive cells was similar in disease and control groups. The increase in caspase 1 positive cells was confirmed by western blotting. Gap density calculated from CLE images correlated significantly with immunohistochemically measured caspase positivity. The authors concluded that CLE measurement of gap density is indeed a valid measure and correlates well with cell death as measured by immunohistochemical caspase staining.

The similarity of caspase 3 positive cells in control and disease subjects is surprising given robust data that TNF is elevated in IBD and that TNF causes apoptotic cell shedding in vivo. The authors speculated that their sample size may have been too small and perhaps there were insufficient patients with active disease to detect differences in caspase 3 staining.

Overall these results are encouraging in that they support the use of CLE as a valid method for evaluating epithelial injury in disease. These data sit well with another recent study that inter-observer and intra-observer agreement is high in CLE and has a relatively short learning curve that does not require prior histological experience [7].

In the future, the utility of CLE is predicted to extend beyond the assessment of cell shedding and barrier loss into the documentation of structural changes such as infiltration by inflammatory cells such as intraepithelial lymphocytes [8]. One intriguing study has used CLE to correlate local epithelial physiological function including barrier function with the gut microbiota overlying that area of epithelium. Bacteria in the genus Bacteroides and Clostridium XIV were inversely correlated with barrier loss [9].

Although all of the studies discussed so far have used intravenous fluorescein as the contrast agent, the development of new fluorescent contrast agents opens the possibility of functional imaging. Topically applied fluorescent anti-TNF antibodies (adalimumab) used in combination with CLE detected membrane-bound TNF-expressing immune cells in the lamina propria [10]. Patients with high numbers of TNF-positive cells have higher response rates to adalimumab than patients with low levels.

Mucosal healing defined as the absence of visible lesions by colonoscopy is a goal of modern therapy for IBD [11]. Should mucosal healing now be defined by the more rigorous standard as the absence of barrier loss and excessive cell shedding as assessed by CLE? Only further clinical trails can answer this question. The development of the probe-based CLE instrument facilitates the collection of CLE images during conventional colonoscopy and requires little experience [7], obviating the need for a dedicated confocal colonoscope, setting the stage for its widespread acceptance, particularly if its clinical benefits are supported by clinical trial data. Nevertheless, it remains to be shown that colonoscopy with or without CLE is superior to simpler and cheaper surrogate markers of mucosal healing such as fecal calprotectin [12]. Certainly, the future is bright for CLE in the diagnosis and assessment of digestive ailments.

\section{References}

1. Marchiando AM, Shen L, Graham WV, et al. The epithelial barrier is maintained by in vivo tight junction expansion during pathologic intestinal epithelial shedding. Gastroenterology. 2011;140:e1-e2. 
2. Kiesslich R, Duckworth CA, Moussata D, et al. Local barrier dysfunction identified by confocal laser endomicroscopy predicts relapse in inflammatory bowel disease. Gut. 2012;61:1146-1153.

3. Rasmussen DN, Karstensen JG, Riis LB, et al. Confocal laser endomicroscopy in inflammatory bowel disease-a systematic review. J Crohns Colitis. 2015;9:1152-1159.

4. Wyatt J, Vogelsang H, Hubl W, et al. Intestinal permeability and the prediction of relapse in Crohn's disease. Lancet. 1993;341:1437-1439.

5. Liu J, Kay T, Davis E, et al. Cell extrusion zones observed on confocal laser endomicroscopy correlate with immunohistochemical staining of mucosal biopsy samples. Dig Dis Sci. (Epub ahead of print). doi:10.1007/s10620-016-4154-x.

6. Liu JJ, Davis EM, Wine E, et al. Epithelial cell extrusion leads to breaches in the intestinal epithelium. Inflamm Bowel Dis. 2013;19:912-921.

7. Chang J, Ip M, Yang M, et al. The learning curve, interobserver, and intraobserver agreement of endoscopic confocal laser endomicroscopy in the assessment of mucosal barrier defects. Gastrointest Endosc. 2016;83:785-795e1.
8. Fritscher-Ravens A, Schuppan D, Ellrichmann M, et al. Confocal endomicroscopy shows food-associated changes in the intestinal mucosa of patients with irritable bowel syndrome. Gastroenterology. 2014;147:1012-1020e4.

9. Wang $\mathrm{AH}, \mathrm{Li} \mathrm{M}, \mathrm{Li} \mathrm{CQ}$, et al. Human colorectal mucosal microbiota correlates with its host niche physiology revealed by endomicroscopy. Sci Rep. 2016;6:21952.

10. Atreya R, Neumann $H$, Neufert $C$, et al. In vivo imaging using fluorescent antibodies to tumor necrosis factor predicts therapeutic response in Crohn's disease. Nat Med. 2014;20:313-318.

11. Schnitzler F, Fidder H, Ferrante M, et al. Mucosal healing predicts long-term outcome of maintenance therapy with infliximab in Crohn's disease. Inflamm Bowel Dis. 2009;15:1295-1301.

12. Lobaton T, Lopez-Garcia A, Rodriguez-Moranta F, et al. A new rapid test for fecal calprotectin predicts endoscopic remission and postoperative recurrence in Crohn's disease. J Crohns Colitis. 2013;7:e641-e651. 